\title{
The diagnostic and prognostic value of angiopoietins compared with C-reactive protein and procalcitonin in children with febrile neutropenia
}

\author{
Esra Mimaroğlu${ }^{1}$, Elvan Çağlar Çıtak ${ }^{1}$, Necdet Kuyucu ${ }^{2}$, Gülçin Eskendari ${ }^{3}$ \\ Departments of ${ }^{1}$ Pediatric Oncology, ${ }^{2}$ Pediatric Infectious Disease, and ${ }^{3}$ Biochemistry, Mersin University Faculty of Medicine, \\ Mersin, Turkey.E-mail: caglarcitak@yahoo.com \\ Received: 14th February 2017, Revised: 29th March 2017, Accepted: 11th April 2017
}

SUMMARY: Mimaroğlu E, Çıtak EÇ, Kuyucu N, Eskendari G. The diagnostic and prognostic value of angiopoietins compared with C-reactive protein and procalcitonin in children with febrile neutropenia. Turk J Pediatr 2017; 59: 418-425.

In this study, we aimed to determine serum angiopoetin (Ang) levels and compare them with levels of C-reactive protein (CRP) and procalcitonin (PCT). Cancer patients (aged 0-18 years) who experienced febrile neutropenia attacks were included in the study. Ang-1, Ang-2, CRP, and PCT were analyzed at admission and $2^{\text {nd }}$ day. Ninety-four episodes of febrile neutropenia that developed in 62 patients were analyzed in this study. The mean age of the patients was $7.56 \pm 4.8(0.5-17)$ years. The patients had lymphoma (33.9\%), solid tumors $(48.4 \%)$, and other cancer $(17.7 \%)$. The percentages of the patients with fever of unknown origin (FUO), clinically documented infection (CDI), and microbiologically documented infection (MDI) categories were $45.7 \%, 22.3 \%$, and $31.9 \%$, respectively. During the study period 11 patients were lost to follow-up. The levels of CRP, PCT and Ang-2 were significantly higher; and that of Ang-1 was significantly lower, compared to the controls.

The differentiation cannot be made between the groups by CRP and PCT levels. The level of Ang-1 was the lowest in MDI group; the level of Ang-2 and the ratio of Ang-2/Ang-1 were high in each group. Ang-1, Ang-2 and the ratio of Ang-2/Ang-1 were significantly associated with mortality. Angs correlated with the severity of infection.

Key words: angiopoietin, children, C-reactive protein, febrile neutropenia, procalcitonin.

Fever is a well-known complication in neutropenic patients with cancer. ${ }^{1}$ During neutropenia, fever may be the first and only clinical manifestation of infection. Because of high morbidity and mortality, evaluation of the source and severity of infection must be fast in a neutropenic patient. To determine the intensity of needed treatments and to decrease the mortality rates, risk classifications were made; and these classifications were based on the measurements of $\mathrm{C}$ - reactive protein (CRP), procalcitonin (PCT), and interleukin 6 (IL-6) biomarkers. ${ }^{2-5}$ CRP and PCT have been reported to be guiding tools for both the prediction of infection and the arrangement of treatment; thus, they are used prevalently. ${ }^{6-8}$ Despite all of these biomarkers, severity of infection and risk of mortality cannot be fully determined. Therefore, new biomarkers have been investigated.9-11

Endothelial barrier disruption plays a key role in the pathogenesis of sepsis and septic shock, making it an attractive target for studies aimed to identify new therapeutic targets for sepsis. ${ }^{12}$ Recently, the participation of VEGF, a vascular growth factor with potent microvascular permeability functions, in the pathogenesis of septic shock was demonstrated. ${ }^{13}$ Another important regulator of endothelial barrier function is the angiopoietins (Ang)- 1 and -2 , and the tyrosine kinase receptor Tie2 expressed in endothelial cells. Binding of Ang-1 to Tie2 maintains the quiescent resting state of the endothelium and reduces vascular permeability in response to inflammatory stimuli. In contrast, 
Ang-2 inhibits biding of Ang-1 to Tie2, resulting in vessel destabilization. ${ }^{14-16}$ Circulating levels of Ang-1 and Ang-2 have been evaluated in patients with sepsis, and levels of Ang-2 have been correlated with sepsis severity in children and adults, when evaluated in patients admitted to intensive care units with established signs and symptoms of sepsis. ${ }^{17-21}$

In this study, we aimed to determine serum Ang-1, Ang-2 levels in febrile neutropenia and differences among febrile neutropenia categories. In addition, we aimed to compare Ang levels with levels of CRP and PCT.

\section{Material and Methods}

Between January 2013 and December 2014, children aged $0-18$ years with cancer were included in this study during their febrile episodes of neutropenia. Characteristics of patients diagnosed with febrile neutropenia such as duration of fever and neutropenia and febrile neutropenia categories were identified, and serum levels of CRP, PCT, Ang-1, and Ang-2 were studied for two separate periods (at admission, at 48th hour of admission).

Febrile neutropenia was defined as the presence of an axillary fever $>38.5^{\circ} \mathrm{C}$ once or $>38^{\circ} \mathrm{C}$ twice within 4 hours in patients with an absolute neutrophil count $<500 / \mathrm{mm}^{3}$ or between $500-1,000 / \mathrm{mm}^{3}$ which is expected to decrease down to $<500 / \mathrm{mm}^{3}$ within $24-$ 48 hours. Fever occurring due to blood or blood product transfusion was excluded. Febrile neutropenia episodes occurring at different times were evaluated as distinct episodes. Patient history was elicited at admission, and a physical examination was performed targeting the foci of infection. Laboratory tests were performed including complete blood count, peripheral blood smear, serum CRP, liver (alanine aminotransferase, aspartate aminotransferase, bilirubin) and renal (urea, creatinine) functional tests, urinalysis and culture, and peripheral blood culture. In addition, catheter samples from catheterized patients were cultured. Cultures were renewed in patients with sustained high fever and bacteremia. In patients with respiratory symptoms or with positive physical examination findings, chest X-rays were obtained. In patients with suspected fungal infections, high-resolution computed chest tomography was performed. In cases with suspected typhlitis and abdominal abscess, advanced radiological imaging techniques such as abdominal ultrasound and tomographic examinations, were carried out.

Blood samples were drawn from patients at admission, and at $48^{\text {th }}$ hours of fever to measure CRP, PCT, Ang-1 and Ang-2 levels. Quantitative determination of hs-CRP in serum by particle enhanced turbidimetric method using INTEGRA 800 plus system (Roche Diagnostics GmbH, D-68298 Mannheim, Germany). Ang-1 levels were measured by ELISA (ng/ml, USCN Life Science Inc, Wuhan, USA, L140801021), Ang-2 levels were measured by ELISA ( $\mathrm{ng} / \mathrm{ml}$, Cusabio Biotech Co, LTD, USA, 222162891).

Febrile neutropenia was divided into three categories according to the severity of infection and febrile neutropenia guidelines: microbiologically documented infection (MDI), clinically documented infection (CDI), and fever of unknown origin (FUO). ${ }^{22}$ MDI was defined as the isolation of microorganism. CDI was considered when there was a focus of infection identified by physical examination without microbiologic documentation. FUO origin was considered when there was no
Ang-1

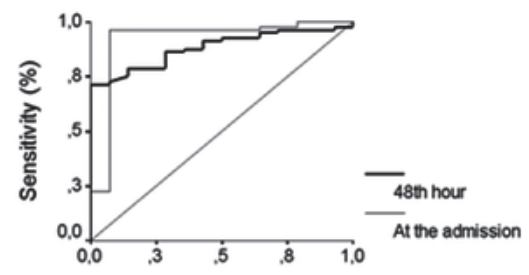

Ang-2

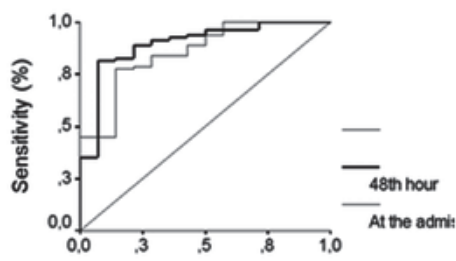

Ang-2/Ang-1 ratio

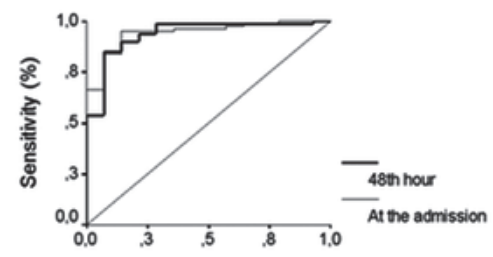

Fig. 1. Receiver operating characteristic ROC curve to determine the accuracy of angiopoietins in prediction mortality at the admission and 48 hour. (A) for Ang-1 levels, (B) for Ang-2 levels and (C) for Ang-2/Ang-1 ratio. 
Table I. Demographic and Laboratory Features of Patients.

\begin{tabular}{lc}
\hline Features & Results \\
\hline Age (year), mean \pm SD (range) & $7.56 \pm 4.8(0.5-17)$ \\
Gender, n (\%) & $34(54.8)$ \\
Male & $28(45.2)$ \\
Female & \\
Primary disease, n (\%) & $21(33.9)$ \\
Non-Hodgkin lymphoma & $8(12.9)$ \\
Neuroblastoma & $6(9.7)$ \\
Osteosarcoma & $5(8.1)$ \\
Ewing sarcoma & $5(8.1)$ \\
Rhabdomyosarcoma & $3(4.8)$ \\
Wilms tumor & $3(4.8)$ \\
Germ cell tumor & $11(17.7)$ \\
Others & \\
Stage, n (\%) & $7(11.3)$ \\
I & $35(56.5)$ \\
II & $20(32.3)$ \\
III & \\
Febrile neutropenia category, $\mathrm{n}(\%)$ & $43(45.7)$ \\
FUO & $21(22.3)$ \\
CDI & $30(31.9)$ \\
MDI & $605 \pm 313$ \\
Leucocyte count $\left(/ \mathrm{mm}^{3}\right)$ & $243 \pm 386$ \\
Absolute neutrophil count $\left(/ \mathrm{mm}^{3}\right)$ & $30 \pm 15$ \\
Absolute monocyte count $\left(/ \mathrm{mm}^{3}\right)$ & $8.37 \pm 1.68$ \\
Hemoglobin (g/dl) & $112,297 \pm 95,082$ \\
Platelet count $\left(/ \mathrm{mm}^{3}\right)$ & \\
\hline CDI: clinically documented infection, FUO: fever of unknown origin, MDI: microbiologically documented infection
\end{tabular}

clinical or microbiologic evidence of infection in a febrile episode. Infections documented both clinically and microbiologically were considered to be microbiologically documented infections. Definite sepsis included sepsis, severe sepsis, and septic shock. Sepsis was defined as systemic inflammatory response syndrome (SIRS) in the presence of or as a result of suspected or proven infection. The definition of severe sepsis is sepsis plus one of the following criteria: cardiovascular organ dysfunction, acute respiratory distress syndrome, or two or more organ dysfunctions (respiratory, renal, neurologic, hematologic, or hepatic). ${ }^{23}$ Levels of Ang-1, Ang-2, CRP, and PCT in various categories of febrile neutropenia, and differences between levels of these parameters in these categories, were investigated.

Following approval of The Ethics Committee of Clinical Research (2013-4 TU) and obtainment of informed consent from the families who volunteered to participate, we began our study.

\section{Statistical Analysis}

Data were analyzed using MedCalc Packet program. The data from this study were evaluated using descriptive statistical methods (mean \pm standard deviation, median, frequencies, and percentages). In the intergroup comparison of categorical variables, a chi-square test was used. Mann Whitney U-test and Kruskal Wallis test were used for comparison of independent variables, and the Wilcoxon test was used for comparison of dependent variables. Spearman's and Pearson correlation coefficient was used to assess relationships between CRP, PCT, Ang-1 and Ang-2 levels. Receiver operator characteristics (ROC) procedures were used to identify optimal cut-off values of Ang concentrations to differentiate patients in groups with FUO, CDI and MDI. A twotailed p value less than 0.05 was considered statistically significant. 


\section{Results}

A total of 94 febrile neutropenia episodes were managed in 62 patients (34 male, 28 female) with a mean age of $7.56 \pm 4.8(0.5-17)$ years; 50 healthy children ( 26 male, 24 female) with a mean age of $6.46 \pm 5.26(0.3-17)$ years during the study period constituted the control group. Demographics and laboratory findings of study group is shown in Table I. Median durations of neutropenia and febrile episode were 11 (3-104) and 6 (1-14) days, respectively. In CDI group 8 patients had mucositis, 3 patients had pneumonia, 2 patients had typhlitis, 2 patients had catheter related infections and 1 patient had varicella zoster infection. In MDI group, there were 29 gram negative infections (most of them Klebsiella, P. aeruginosa and E. coli), 22 gram positive infections (especially coagulase negative staphylococci) and 9 candida infections. Of the 94 episodes of FN, 20 (21.2\%) evolved to septic shock, and 11 (11.7\%) were fatal. All deaths were attributed to complications of septic shock, and occurred after a median of 11 days (range 3 to 28) from sample collection.

The mean CRP, PCT, Ang-1, and Ang-1 levels and Ang-2/Ang-1 ratio at admission and at $48^{\text {th }}$ hours in FUO, CDI and MDI groups and comparison between groups are shown in Table
II. A positive correlation was found between platelet count and Ang-1 levels $(r=0.77$, $\mathrm{p}=0.001$ ).

When the relations between CRP, PCT levels and mortality were examined, it was determined that there was no significant difference between them (Table III). Ang-1 levels were significantly lower and Ang-2 levels were significantly higher in non-survivors, compared to survivors, both at admission and at $48^{\text {th }}$ hours $(\mathrm{p}<0.001$; Table III). Threshold was determined to the usability of determining the mortality of the all parameters. ROC analyses ad sensitivity, specificity, positive and negative predictive values of Ang-1, Ang-2 and Ang-2/Ang-1 ratio at specific values are presented in Figure 1 and Table IV.

\section{Discussion}

Post chemotherapy FN in patients with hematological malignancies is a condition that carries a high risk of sepsis complications with mortality rates as high as $21 \%$, usually preceded by septic shock. ${ }^{24}$ Patients with FN are a heterogeneous group in terms of risks of complications and mortality, and the identification of parameters capable to accurately identify high risk patients is one of the great PCT have been recently proved to be

Table II. CRP, Procalcitonin, Angiopoetin-1, Angiopoetin-2, Angiopoetin-2/Angiopoetin-1 Test Results in Group of Febrile Neutropenia.

\begin{tabular}{|c|c|c|c|c|c|}
\hline & $\begin{array}{c}\text { CRP } \\
(\mathrm{mg} / \mathrm{L})\end{array}$ & PCT (ng/ml) & $\begin{array}{l}\text { Ang-1 } \\
(\mathrm{ng} / \mathrm{ml})\end{array}$ & $\begin{array}{l}\text { Ang-2 } \\
(\mathrm{ng} / \mathrm{ml})\end{array}$ & $\begin{array}{c}\text { Ang-2/Ang- } 1 \\
\text { ratio }\end{array}$ \\
\hline \multicolumn{6}{|l|}{ FUO } \\
\hline At the admission & $46.19 \pm 61.24$ & $2.39 \pm 2.22$ & $1.33 \pm 0.49$ & $3.52 \pm 3.02$ & $3.62 \pm 5.49$ \\
\hline $4^{\text {th }}$ hour & $65.64 \pm 70.49$ & $1.69 \pm 0.96$ & $1.79 \pm 4.02$ & $1.97 \pm 2.14$ & $5.31 \pm 10.57$ \\
\hline \multicolumn{6}{|l|}{ CDI } \\
\hline At the admission & $74.24 \pm 55.04$ & $2.58 \pm 1.89$ & $1.14 \pm 0.49$ & $6.59 \pm 2.52$ & $8.61 \pm 8.79$ \\
\hline $48^{\text {th }}$ hour & $92.20 \pm 49.62$ & $2.44 \pm 7.38$ & $0.93 \pm 0.54$ & $5.12 \pm 2.13$ & $8.24 \pm 6.91$ \\
\hline \multicolumn{6}{|l|}{ MDI } \\
\hline At the admission & $101.84 \pm 72.90$ & $10.51 \pm 7.87$ & $0.64 \pm 0.40$ & $12.63 \pm 3.99$ & $31.48 \pm 30.96$ \\
\hline $48^{\text {th }}$ hour & $131.03 \pm 81.94$ & $8.95 \pm 11.46$ & $0.40 \pm 0.29$ & $10.67 \pm 4.01$ & $51.48 \pm 51.93$ \\
\hline $\mathrm{p}^{1}$ & 0.04 & 0.063 & 0.313 & $<0.0001$ & $<0.0001$ \\
\hline $\mathrm{p}^{2}$ & $<0.0001$ & $<0.0001$ & $<0.0001$ & $<0.0001$ & $<0.0001$ \\
\hline $\mathrm{p}^{3}$ & 0.063 & $<0.0001$ & $<0.001$ & $<0.0001$ & $<0.0001$ \\
\hline $\mathrm{p}^{4}$ & 0.01 & 0.076 & 0.488 & $<0.0001$ & $<0.001$ \\
\hline $\mathrm{p}^{5}$ & $<0.0001$ & $<0.0001$ & $<0.0001$ & $<0.0001$ & $<0.0001$ \\
\hline $\mathrm{p}^{6}$ & 0.072 & $<0.0001$ & $<0.0001$ & $<0.0001$ & $<0.0001$ \\
\hline
\end{tabular}

Ang-1: angiopoietin-1, Ang-2: angiopoietin-2, CDI: clinically documented infection, CRP: C-reactive protein, MDI: microbiologically documented infection, FUO: fever of unknown origin, PCT: procalcitonin, $\mathrm{p}^{1}$ : FUO vs CDI at admission, $\mathrm{p}^{2}$ : FUO vs MDI at admission, $\mathrm{p}^{3}$ : CDI vs MDI at admission, $\mathrm{p}^{4}$ : FUO vs CDI at $48^{\text {th }}$ hour, $\mathrm{p}^{5}$ : FUO vs MDI at $48^{\text {th }}$ hour, $\mathrm{p}^{6}$ : CDI vs MDI at $48^{\text {th }}$ hour 
Table III. CRP, Procalcitonin, Angiopoetin-1, Angiopoetin-2, Angiopoetin-2/Angiopoetin-1 Test Results in Survivor and Non-survivor Patients.

\begin{tabular}{lccc}
\hline & $\begin{array}{c}\text { Survivors } \\
(\mathrm{n}=52)\end{array}$ & $\begin{array}{c}\text { Non-survivors } \\
(\mathrm{n}=11)\end{array}$ & p value \\
\hline CRP $(\mathrm{mg} / \mathrm{L})$ at the admission & $67.97 \pm 69.92$ & $83.08 \pm 54.43$ & 0.121 \\
$\mathrm{CRP}(\mathrm{mg} / \mathrm{L})$ at the $48^{\text {th }}$ hour & $86.98 \pm 72.13$ & $123.66 \pm 88.23$ & 0.104 \\
PCT $(\mathrm{ng} / \mathrm{ml})$ at the admission & $4.79 \pm 6.79$ & $6.62 \pm 5.25$ & 0.066 \\
PCT $(\mathrm{ng} / \mathrm{ml})$ at the $48^{\text {th }}$ hour & $3.93 \pm 8.18$ & $6.76 \pm 11.47$ & 0.057 \\
Ang-1 $(\mathrm{ng} / \mathrm{ml})$ at the admission & $1.18 \pm 0.50$ & $0.41 \pm 0.33$ & $<0.0001$ \\
Ang-1 $(\mathrm{ng} / \mathrm{ml})$ at the $48^{\text {th }}$ hour & $1.32 \pm 2.99$ & $0.22 \pm 0.13$ & $<0.0001$ \\
Ang-2 $(\mathrm{ng} / \mathrm{ml})$ at the admission & $6.00 \pm 4.23$ & $13.44 \pm 5.38$ & $<0.0001$ \\
Ang-2 $(\mathrm{ng} / \mathrm{ml})$ at the 48 $48^{\text {th }}$ hour & $4.29 \pm 3.65$ & $12.03 \pm 5.01$ & $<0.0001$ \\
Ang-2/Ang-1 ratio at the admission & $7.87 \pm 10.26$ & $46.49 \pm 38.15$ & $<0.0001$ \\
Ang-2/Ang-1 ratio at the 48 ${ }^{\text {th }}$ hour & $10.30 \pm 20.08$ & $80.09 \pm 52.50$ & $<0.0001$ \\
\hline
\end{tabular}

Ang: angiopoetin, CRP: C-reactive protein, PCT: procalcitonin

useful tools for this purpose. ${ }^{25}$ However, it is generally acknowledged that these parameters are more useful to identify patients at low risk for complications. There are limited studies evaluating importance of CRP and proinflammatory cytokine levels in detecting bacterial infection and clinical progress during febrile neutropenia attack.

Santolaya et al. ${ }^{26}$ showed a CRP value of $>40 \mathrm{mg} / \mathrm{L}$ discriminated children with a demonstrated bacterial infection (sensitivity $100 \%$, specificity $76.6 \%$ ) from children with febrile neutropenia. They also showed that children with an unfavorable outcome had persistently high levels of serum CRP. Rintala et al. ${ }^{27}$ found that CRP levels were significantly higher when the blood culture was positive and CRP levels were significantly higher when the infection focus was identified. They concluded that the serial measurement of CRP is a reliable and readily available means for differentiating between bacterial infections and other causes of fever in patients with hematological malignancies, during neutropenia and after cytotoxic treatment. Mian et al. ${ }^{28}$ showed that CRP $>100 \mathrm{mg} / \mathrm{L}$ had a sensitivity of $88 \%$ in predicting high-risk FN.

Sakr el al. ${ }^{29}$ reviewed 30 articles which investigated the PCT levels in FN. They found that PCT seems to be able to discriminate fever due to systemic types of infection from non-infectious etiologies. They also found that PCT might be useful in outcome prediction in patients with FN but it was not superior to CRP concentration. Engel et al. ${ }^{30}$ detected an increase in PCT levels in the first 32 hours of fever in patients with clinically or microbiologically proven infection. They also found that PCT levels were lower in patients with fever of unknown origin when compared with proven infection. Karakurt DG et al. ${ }^{2}$ did not find IL-6, IL-8, sTNFRII, sIL-2R, CRP, and PCT levels as significant predictors of serious infectious complication in pediatric cancer patients' admission for febrile neutropenia. The concentration of lipopolysaccharidebinding protein (LBP), PCT, interleukin-6, and CRP was determined for 90 episodes of FN by Kitanovski et al. ${ }^{31}$ The concentrations of all biomarkers were significantly higher in patients with bacteremia/clinical sepsis than non-bacteremia/clinical sepsis episodes on admission and day 2. The diagnostic accuracy of PCT did not significantly different from CRP. A recent meta-analysis showed that the PCT test has higher diagnostic value than CRP or IL- 6 for the detection of bacterial infection in patients with FN. In that study, data suggested that PCT is a more specific than sensitivity and helps to confirm bacterial infections rather than rule them out. 32

Our results showed that a lowest CRP level was found in FUO group. According to CRP levels we could not distinguished the CDI and MDI group in this study both at the admission and at $48^{\text {th }}$ hours. In our study CRP levels were not statistically different in survived and non-survived patients. CRP was not a useful 
marker for detecting the mortality according to our study. These results are similar to the literature. ${ }^{10,11}$ We could not distinguish FUO and CDI according to PCT levels. The highest level of PCT was found in MDI group in our study. These results showed that PCT is more useful, compared to CRP, to discriminate the MDI group. Our results also showed that PCT levels (both at admission and at the $48^{\text {th }}$ hour) were helpful to predict bacteremia. These results are similar to the literature. ${ }^{10,11}$ However, PCT levels were not helpful to predict the mortality.

In the developed vasculature, Ang- 1 protects against vascular leakage, whereas Ang-2 promotes increased vascular permeability. Ang-1 and Ang-2 appear an agonist-antagonist effect to pair mediating capillary endothelium leakage (i.e., Ang-2/Ang-1). The higher the Ang-1/Ang-2 ratio is, the more severe the capillary endothelial damage. In addition, Ang- 1 has been proposed to be necessary for the maintenance of vascular barrier function via its interaction with Tie-2 in blood vessels. As a default pathway, constitutive Ang-1-Tie-2 interactions promote endothelial cell migration, proliferation, survival, and differentiation and regulate endothelial barrier integrity and stability. ${ }^{11,33}$ A lower Ang-1/Tie-2 ratio indicates a stronger defense against capillary endothelial damage. Taken together, the increase in the Ang-2/Ang-1 ratio and the decrease in the Ang- $1 /$ Tie- 2 ratio demonstrate that destruction of the capillary endothelium is greater than the stability in the deterioration of sepsis. Thus, high admission serum Ang-2/Ang-1 ratio and low admission serum Ang-1/Tie-2 ratio identify the severity of sepsis in patients at the onset of early sepsis in the emergency department. ${ }^{34}$ Increased levels of Ang-2 levels have been demonstrated in adult and pediatric patients with sepsis in intensive care units (ICU) and these levels correlated with sepsis severity. ${ }^{17,18,35}$ Serum obtained from patients with sepsis has been shown to disrupt endothelial architecture, an effect that correlates with Ang-2 levels, is reversed by Ang-1.36

Kumpers et al. ${ }^{18}$, studying a population of 43 medical ICU patients demonstrated that an Ang-2 level at the time of ICU admission was a strong predictor of mortality. Ang-1 levels were also evaluated in some of these studies, but so far studies have failed to demonstrate a relationship between Ang-1 and sepsis outcomes.

To our knowledge, there were 2 studies about FN and angiopoietins in adult population. 10,11 Our study is the first one with neutropenic fever attacks in childhood. Our results suggest that the decrease in the level of Ang-1 occurs to protect the damage of the endothelial barrier in MDI group. The results at $48^{\text {th }}$ hour are similar to that at the admission: the level of Ang-2 is significantly higher in MDI and CDI group, compared to FUO group. We may think that the increase in the level of Ang-2 is related to damage in the endothelial barrier. Moreover, it has a role as a distinctive feature among FUO, CDI and MDI groups and has a positive correlation with the severity of the infection and this could be a distinctive parameter for

Table IV. Specificity, Sensitivity, Positive Predictive Value (PPV), Negative Predictive Value (NPV), and Diagnostic Performance of Angiopoietin (Ang)-1, Ang-2 Concentrations and Ang-2/Ang-1 Ratio According to Cutoff Values at the Admission and $48^{\text {th }}$ Hour for Predicting Mortality.

\begin{tabular}{lccccc}
\hline & $\begin{array}{c}\text { Specificity } \\
(\%)\end{array}$ & $\begin{array}{c}\text { Sensitivity } \\
(\%)\end{array}$ & $\begin{array}{c}\text { PPV } \\
(\%)\end{array}$ & $\begin{array}{c}\text { NPV } \\
(\%)\end{array}$ & $\begin{array}{c}\text { Diagnostic } \\
\text { Performance }\end{array}$ \\
\hline At the admission & & & & & \\
$\quad$ Ang-1 $(\leq 0.452 \mathrm{ng} / \mathrm{ml})$ & 96.2 & 92.6 & 81.2 & 98.7 & 0.920 \\
Ang-2 $(>9.168 \mathrm{ng} / \mathrm{ml})$ & 77.5 & 85.7 & 40.0 & 96.9 & 0.854 \\
$\quad$ Ang-2/Ang-1 $(>21.71)$ & 95.0 & 85.8 & 75.0 & 97.4 & 0.942 \\
48 & & & & & \\
Ang-1 $(\leq 0.436 \mathrm{ng} / \mathrm{ml})$ & 71.2 & 100.0 & 37.8 & 100.0 & 0.881 \\
Ang-2 $(>7.675 \mathrm{ng} / \mathrm{ml})$ & 81.2 & 92.9 & 46.4 & 98.5 & 0.896 \\
Ang-2/Ang-1 $(>17.32)$ & 85.0 & 92.9 & 52.0 & 98.6 & 0.937 \\
\hline
\end{tabular}


these groups but needs to be supported by other studies.

In adult studies, only ratio of Ang-2 to Ang-1 were found predictive parameters for mortality. ${ }^{10,11}$ Contrary to the data obtained from studies in adults, both Ang-1, Ang-2 and the ratio of Ang-2 to Ang-1 had determinant effects on mortality in our study. In the current study, the sensitivity, specificity, positive and negative predictive value of Ang-1, Ang-2 and the ratio of Ang-2 to Ang-1 were comparable. We assume that the difference of the data in adults is due to the immaturity of the endothelial barrier in childhood. But as it is the first study about FN in childhood, the results have to be supported by the further studies.

In conclusion, our study suggests that Ang-1, Ang-2, and Ang-2/Ang-1 ratio are biomarkers for 28-day mortality in childhood FN.

\section{Acknowledgements}

We would like to thank Prof. Dr. Bahar Tasdelen for the data analysis. This project was supported by Mersin University Department of Scientific Research Projects (Project No: 2013-4 TU and The Scientific and Technological Research Council of Turkey (TUBITAK) 1002 Short Term R\&D Funding Program (Project No: 114S061).

\section{REFERENCES}

1. Castagnola E, Fontana V, Caviglia I, et al. A prospective study on the epidemiology of febrile episodes during chemotherapy-induced neutropenia in children with cancer or after hemopoietic stem cell transplantation. Clin Infect Dis 2007; 45: 1296-1304.

2. Karakurt DG, Demirsoy U, Corapcioglu F, et al. Do proinflammatory cytokine levels predict serious complication risk of infection in pediatric cancer patients? Pediatr Hematol Oncol 2014; 31: 415-424.

3. Phillips RS, Wade R, Lehrnbecher T, et al. Systematic review and meta-analysis of the value of initial biomarkers in predicting adverse outcome in febrile neutropenic episodes in children and young people with cancer. BMC Med 2012; 10: 6.

4. Semeraro M, Thomée C, Rolland E, et al. A predictor of unfavourable outcome in neutropenic paediatric patients presenting with fever of unknown origin. Pediatr Blood Cancer 2010; 54: 284-290.

5. HaeuslerGM, Carlesse F, Phillips RS. An updated systematic review and meta-analysis of the predictive value of serum biomarkers in the assessment of fever during neutropenia in children with cancer. Pediatr Infect Dis J 2013; 32: e390-e396.
6. Kim DY, Lee YS, Ahn S, et al. The usefulness of procalcitonin and C-reactive protein as early diagnostic markers of bacteremia in cancer patients with febrile neutropenia. Cancer Res Treat 2011; 43: 176-180.

7. Ahn S, Lee YS. Predictive factors for poor prognosis febrile neutropenia. Curr Opin Oncol 2012; 24: 376380.

8. von Lilienfeld-Toal M, Dietrich MP, Glasmacher A, et al. Markers of bacteremia in febrile neutropenic patients with hematological malignancies: procalcitonin and IL-6 are more reliable than C-reactive protein. Eur J Clin Microbiol Infect Dis 2004; 23: 539-544.

9. Mian A, Becton D, Saylors R, et al. Biomarkers for risk stratification of febrile neutropenia among children with malignancy: a pilot study. Pediatr Blood Cancer 2012; 59: 238-245.

10. Luz Fiusa MM, Costa-Lima C, de Souza GR, et al. A high angiopoetin2/angiopoetin1 ratio is associated with a high risk of septic shock in patients with febrile neutropenia. Crit Care 2013; 17: R169.

11. Alves BE, Montalvao SA, Aranha FJ, et al. Imbalances in serum angiopoetin concentrations are early predictors of septic shock development in patients with post chemotherapy febrile neutropenia. BMC Infect Dis 2010; 10: 143.

12. Spronk PE, Zandstra DF, Ince C. Bench-to-bedside review: Sepsis is a disease of the microcirculation. Crit Care 2004; 8: 462-468.

13. Yano K, Liaw PC, Mullington JM, et al. Vascular endothelial growth factor is an important determinant of sepsis morbidity and mortality. J Exp Med 2006; 203: $1447-1458$.

14. Fiedler U, Reiss Y, Scharpfenecker $M$, et al. Angiopoietin-2 sensitizes endothelial cells to TNF-alpha and has a crucial role in the induction of inflammation. Nat Med 2006; 12: 235-239.

15. Maisonpierre PC, Suri C, Jones PF, et al. Angiopoietin-2, a natural antagonist for Tie 2 that disrupts in vivo angiogenesis. Science 1997; 277: 55-60.

16. Thurston G, Rudge JS, Ioffe E, et al. Angiopoietin-1 protects the adult vasculature against plasma leakage. Nat Med 2000; 6: 460-463.

17. Giuliano JS Jr, Lahni PM, Harmon K, et al. Admission angiopoietin levels in children with septic shock. Shock 2007; 28: 650-654.

18. Kumpers P, Lukasz A, David S, et al. Excess circulating angiopoietin-2 is a strong predictor of mortality in critically ill medical patients. Crit Care 2008; 12: R147.

19. Kumpers P, van Meurs M, David S, et al. Time course of angiopoietin-2 release during experimental human endotoxemia and sepsis. Crit Care 2009; 13: R64.

20. Parikh SM, Mammoto T, Schultz A, et al. Excess circulating angiopoietin-2 may contribute to pulmonary vascular leak in sepsis in humans. PLoS Med 2006; 3: e46.

21. Siner JM, Bhandari V, Engle KM, et al. Elevated serum angiopoietin 2 levels are associated with increased mortality in sepsis. Shock 2009; 31: 348-353. 
22. Freifeld AG, Bow EJ, Sepkowitz KA, et al. Infectious Diseases Society of America. Clinical practice guideline for the use of antimicrobial agents in neutropenic patients with cancer: 2010 Update by the Infectious Diseases Society of America. Clin Infect Dis 2011; 52: 427-431.

23. Goldstein B, Giroir A, Randolph A. An international consensus conference on Pediatric sepsis. International pediatric sepsis consensus conference: Definitions for sepsis and organ dysfunction in pediatrics. Pediatr Crit Care Med 2005; 6: 2-8.

24. Herbst C, Naumann F, Kruse EB, et al. Prophylactic antibiotics or G-CSF for the prevention of infections and improvement of survival in cancer patients undergoing chemotherapy. Cochrane Database Syst Rev 2009: CD007107.

25. Ellis M. Febrile neutropenia. Ann N Y Acad Sci 2008; 1138: 329-350.

26. Santolaya ME, Cofre J, Beresi V. C-reactive protein: A valuable aid for the management of febrile children with cancer and neutropenia. Clin Infect Dis 1994; 18: 589-595.

27. Rintala E, Irjala K, Nikoskelainen J. Value of measurement of C-reactive protein in febrile patients with hematological malignancies. Eur J Clin Microbiol Infect Dis 1992; 11: 973-978.

28. Mian A, Becton D, Saylors R, et al. Biomarkers for risk stratification of febrile neutropenia among children with malignancy: A pilot study. Pediatr Blood Cancer 2012; 59: 238-245.

29. Sakr Y, Sponholz C, Tuche F, et al. The role of procalcitonin in febrile neutropenic patients: Review of the literature. Infection 2008; 36: 396-407.
30. Engel A, Steinbach G, Kern P, et al. Diagnostic value of procalcitonin serum levels in neutropenic patients with fever: Comparison with interleukin-8. Scand J Infect Dis 1999; 31: 185-189.

31. Kitanovski L, Jazbec J, Hojker S, et al. Diagnostic accuracy of lipopolysaccharide-binding protein for predicting bacteremia/clinical sepsis in children with febrile neutropenia: Comparison with interleukin-6, procalcitonin, and C-reactive protein. Support Care Cancer 2014; 22: 269-277.

32. Wu CW, Wu JY, Chen $\mathrm{CK}$, et al. Does procalcitonin, C-reactive protein, or interleukin-6 test have a role in the diagnosis of severe infection in patients with febrile neutropenia? A systematic review and metaanalysis. Support Care Cancer 2015; 23: 2863-2872.

33. Mofarrahi M, Nouh T, Qureshi S, et al. Regulation of angiopoietin expression by bacterial lipopolysaccharide. Am J Physiol Lung Cell Mol Physiol 2008; 294: L955-L963.

34. Fang Y, Li C, Shao R, et al. Prognostic significance of the angiopoietin -2/angiopoietin-1/Tie-2 ratios for early sepsis in an emergency department. Crit Care 2015; 19: 367.

35. Wang K, Bhandari V, Giuliano JS Jr, et al. Angiopoietin-1, angiopoietin-2, and bicarbonate as diagnostic biomarkers in children with severe sepsis. PLoS One 2014; 9: e108461.

36. Parikh SM, Mammoto T, Schultz A, et al. Excess circulating angiopoietin-2 may contribute to pulmonary vascular leak in sepsis in humans. PLoS Med 2006; 3: e46. 